\title{
Molecular characterization of a novel PAX9 missense mutation causing posterior tooth agenesis
}

\author{
Hitesh Kapadia $^{1,2}$, Sylvia Frazier-Bowers ${ }^{1,3}$, Takuya Ogawa ${ }^{1}$ and Rena N D'Souza ${ }^{*, 1}$ \\ ${ }^{1}$ Department of Orthodontics, Dental Branch, University of Texas at Houston Health Science Center, Houston, \\ TX 77030, USA; ${ }^{2}$ Program in Genes and Development, Graduate School of Biomedical Sciences (GSBS), University \\ of Texas at Houston Health Science Center and MD Anderson Cancer Center, Houston, TX 77030, USA
}

Autosomal dominant mutations in the gene encoding the paired box containing transcription factor PAX9 are associated with nonsyndromic human tooth agenesis that primarily affect posterior dentition. The molecular mechanisms contributing to its pathogenesis are poorly understood. In this study, we describe a novel mutation in PAX9 in a family with molar oligodontia. This heterozygous mutation results in the substitution of a highly conserved isoleucine residue by phenylalanine within the carboxyl-terminal subdomain of the paired domain. Immunolocalization and cell fractionation studies to ascertain the subcellular localization of the Ile87Phe protein showed that both wild-type and mutant proteins are synthesized in mammalian cells and that the mutation does not alter the nuclear localization of the mutant protein. Gel-shift assays using two cognate paired-domain recognition sequences, e5 and CD19-2(A-ins), revealed that while wild-type Pax9 binds to both sequences, the mutant protein was unable to bind these sites. In addition, the latter did not alter the DNA-binding activities of wild-type Pax9. Furthermore, we evaluated the ability of the Ile87Phe mutant protein to form a complex with a partner protein, Msx1, and found that the mutation under study has no effect on this interaction. Based on our observed defects in DNA binding by the mutant protein, we propose a loss-of-function mechanism that contributes to haploinsufficiency of PAX9 in this family with posterior tooth agenesis.

European Journal of Human Genetics (2006) 14, 403-409. doi:10.1038/sj.ejhg.5201574; published online 15 February 2006

Keywords: oligodontia; Pax9; missense mutation; Msx1

\section{Introduction}

Studies of the developing mouse tooth germ have yielded significant insights into the genetics and molecular mechanisms of mammalian tooth development. ${ }^{1,2}$ Prominent among these has been the identification of two transcription factors, Pax9 and Msx1, which play fundamental

\footnotetext{
*Correspondence: Dr RN D'Souza, Department of Orthodontics, Dental Branch, Room 346, University of Texas Health Science Center, 6516 MD Anderson Boulevard, Houston, TX 77030, USA. Tel: + 1713500 4218; Fax: + 1713500 4123; E-mail: rena.n.dsouza@uth.tmc.edu

${ }^{3}$ Current address: Department of Orthodontics, School of Dentistry, University of North Carolina at Chapel Hill, Chapel Hill, NC 27599-7450, USA.

Received 15 July 2005; revised 16 November 2005; accepted 7 December 2005; published online 15 February 2006
}

roles in epithelial-mesenchymal interactions during odontogenesis. Both are developmental regulatory molecules that are required during normal tooth development. $^{1-3}$ Furthermore, human genetics studies have uncovered distinct patterns of nonsyndromic posterior tooth agenesis (oligodontia (MIM 604625)) associated with defects in the two genes. Despite these advances, there remain gaps in our current understanding of the molecular pathogenesis of this disorder.

Nonsyndromic familial tooth agenesis is a common developmental anomaly of dentition characterized by one or more congenitally missing teeth. The unavoidable dental consequences include malocclusion because of improper position of the teeth, deficient growth of the alveolar processes associated with the missing teeth, ${ }^{4}$ and 
excess space within the dental arches. Since the initial discovery of an insertion mutation within the paired domain of $P A X 9,{ }^{5}$ a spectrum of autosomal dominant mutations ranging from missense to premature stops have been associated with the disorder. ${ }^{6}$ The mutations are spread throughout the entire PAX9 gene with clustering in the paired DNA-binding domain. Predictably, many would alter the resultant protein products to an extent that would render them nonfunctional. ${ }^{5,7-11}$ Others are suggestive of haploinsufficiency that can be attributed to deletion of an allele of PAX9. ${ }^{12}$ Recently, a loss-of-function mechanism has been proposed for a substitution mutation within the amino-terminal (N-terminal) subdomain of the paired domain that results in a reduction of DNA-binding affinity. ${ }^{13}$ Despite these advances, the precise mechanisms by which other PAX9 mutations, specifically those within the paired domain, affect the phenotype are not clearly defined.

Here we report a novel mutation identified in a family with nonsyndromic autosomal dominant tooth agenesis and investigate its biochemical and functional consequences through an analysis of the mutant protein derived from a mouse homologue. Our functional analysis revealed an abrogation of binding by the mutant protein to two cognate-paired domain recognition sequences. The absence of other defects at the protein level suggests that the disruption of the DNA-binding mechanism underlies the pathogenesis observed in this family with tooth agenesis.

\section{Materials and methods}

\section{Clinical evaluation and pedigree analysis}

The family was first brought to our attention by a medical geneticist at Albert Einstein Medical Center in Pennsylvania. The index case was a 15-year-old female (III:1) with one affected sibling (III:2, 19 years) and an affected father (II:1, 54 years) and paternal grandfather (I:1, 83 years). The affected father had no siblings or cousins and therefore was the only affected individual in his generation. Detailed family and medical histories were taken based on a custom questionnaire and panoramic radiographs and/or intraoral photos were provided by the family dentist. Clinical evaluation included a documentation of the teeth present, as well as a notation of obvious morphological anomalies such as peg-shaped teeth. This study was approved by the University of Texas Health Science Center at Houston Committee for the Protection of Human Subjects. Consent to participate in this study (including a release for dental records) was obtained from every adult participant, or a parental guardian in the case of minors.

\section{Sequencing and mutational analysis}

Peripheral blood samples or buccal swabs were obtained for five members of the family, which include three generations (four affected and one unaffected). DNA extractions were performed for all family members using Puregene DNA isolation kit (Gentra Systems, Minneapolis, MN, USA), followed by amplification using polymerase chain reaction (PCR) with primer sets for exons 1-4 of PAX9 and amplification conditions as previously described, ${ }^{9}$ and purification with ExoSap-IT reagent (USB, Cleveland, $\mathrm{OH}$, USA). Direct sequencing of the coding regions, including intron-exon boundaries was performed using ABI Big Dye $^{\circledR}$ terminator reagents (Applied Biosystems, Foster City, CA, USA) and an ABI PRISM 3730 DNA sequencer. Sequences were compared using NCBI Blast2 software (http://www.ncbi.nlm.nih.gov/entrez/query.fcgi) with EMBL entries AJ238381, AJ238382 and AJ238383. Additionally, 100 unrelated control individuals were sequenced.

Construction of GST-fused expression plasmids pGEXPax9 and pGEX-A259T Briefly, a 2.4-kb murine Pax9 cDNA clone comprising the full-length coding sequence and portions of both the $5^{\prime}$ - and $3^{\prime}$-untranslated regions that was originally provided by Dr Annette Neubüser (Research Institute of Molecular Pathology, Wien, Austria) was used to construct the pGEX-Pax9 expression vector as previously described. ${ }^{7}$ To construct pGEX-A259TPax9, in vitro site-directed mutagenesis was performed using the QuikChange mutagenesis kit (Stratagene, CA, USA).

\section{Construction of mammalian expression plasmids}

The mammalian expression vector pCMV-Pax9 with c-Myc epitope tag is previously described., ${ }^{74}$ The pCMVA259TPax9 expression vector was constructed in the same manner, using the full-coding sequence of A259TPax9 cDNA (obtained from BamHI-SalI restriction digest of pGEX-A259T Pax9). Sequencing was performed to verify the c-Myc epitope was in frame with the N-terminus of mutant Pax9. The expression plasmid containing the CMV promoter linked to full-coding sequence of Msx1 was constructed in pCMV-Tag2b (Stratagene, CA, USA).

Purification of GST-Pax9 and GST-Ile87Phe Pax9 fusion proteins GST-fusion proteins were generated and purified as previously described. ${ }^{7}$

Electrophoretic mobility shift assay Oligonucleotides corresponding to e5 and CD19-2(A-ins) were synthesized (Sigma/Genosys, TX, USA) and gel retardation assays were performed as previously described. ${ }^{7}$ The oligonucleotide probes are two previously established high-affinity paired domain-binding sites. The e5 sequence was originally derived from the Drosophila even skipped promoter for the Eve transcription factor. ${ }^{15}$ Several Pax proteins, including Pax9, are known to bind to this sequence. ${ }^{16}$ CD19-2(A-ins) was chosen as it is a classical paired domain recognition sequence. $^{14}$ Initially, radiolabeled e5 or CD19-2(A-ins) was incubated with $100 \mathrm{ng}$ of affinity-purified GST-Pax9 and GST-Ile87Phe proteins. To detect dominant-negative 
activity of the mutant protein, a fixed amount of GST-Pax9 (200 ng) was added to increasing amounts (200, 400, $600 \mathrm{ng}$ ) of GST-Ile87Phe Pax9. Electrophoretic mobility shift assay (EMSA) was performed using the CD19-2(A-ins) probe. In all cases, EMSA was performed as previously described $^{7}$ and in triplicate.

\section{Subcellular localization of wild-type Pax9 and mutant} Ile87Phe Pax9 in mammalian cells

To demonstrate the in vivo expression of wild-type and mutant Pax9, COS7 cells were obtained from American Type Culture Collection (ATTC) and grown as described previously. ${ }^{7}$ They were transfected with c-Myc-Pax9 or c-Myc-A259TPax9 using FuGENE 6 (Roche Molecular Biochemicals, IN, USA) according to the manufacturer's instructions. Subcellular localization of wild-type and mutant Pax9 proteins was shown by immunolocalization studies at $48 \mathrm{~h}$ after transfection. Transfected cells were fixed and permeabilized with $-10^{\circ} \mathrm{C}$ methanol for $5 \mathrm{~min}$. Cells were incubated for $1 \mathrm{~h}$ with c-Myc antibody at 1:100 dilution, and washed and incubated with a fluorescein isothiocyanate-conjugated goat anti-mouse antibody (Santa Cruz Biotechnology, CA, USA) to visualize c-Myc. Cells were examined with an Olympus $\mathrm{BH}-2$ microscope.

To confirm our immunocytochemistry results, nuclear and cytoplasmic fractions were obtained from cells transfected for $48 \mathrm{~h}$ using the Nuclear Extract Kit (Active Motif, CA, USA) according to manufacturer's instructions. Western blotting was then performed using a previously established protocol. ${ }^{7}$

\section{Co-immunoprecipitation}

COS7 cells were grown as described previously ${ }^{7}$ and cotransfected with c-Myc-Pax9 or cMyc-A259TPax9 and FLAG-Msx1 using FuGENE 6 (Roche Molecular Biochemicals, IN, USA) according to the manufacturer's instructions. After $48 \mathrm{~h}$, cells were resuspended in lysis buffer (50 mm Tris, pH 8.0, $400 \mathrm{~mm} \mathrm{NaCl,} \mathrm{1 \%} \mathrm{Triton} \mathrm{X-100)} \mathrm{with}$ protease inhibitor cocktail tablets (Roche) and incubated for $30 \mathrm{~min}$ on ice. Cell lysates were added to $20 \mu \mathrm{l}$ of antiFLAG M2 affinity gel (Sigma) and rotated at $4{ }^{\circ} \mathrm{C}$ overnight. The affinity gels were washed with lysis buffer four times and TBS once and eluted with Laemmli buffer, and analyzed by Western blotting with 1:200 dilution anti-cMyc (9E10) monoclonal antibody (Santa Cruz Biotechnology) or 1:1000 dilution mouse anti-FLAG M2 monoclonal antibody (Sigma) using ECL kit (Pharmacia Amersham Biotech).

\section{Results}

Identification of an adenine to thymine transversion in a family with posterior tooth agenesis

We report a phenotype that includes agenesis of permanent posterior teeth in one family that shows an autosomal dominant inheritance pattern. A detailed clinical examination revealed a pattern of tooth agenesis similar to those previously published where molars were predominantly absent (Figure 1). The affected father and paternal grandfather, who both reported multiple missing molars, are omitted from the table as dental records were not available. All family members were otherwise medically healthy, and revealed no apparent abnormalities of other organ systems. Sequence analysis of the affected siblings, father and grandfather revealed a heterozygous $\mathrm{A}$ to $\mathrm{T}$ transversion mutation at nucleotide 259 in exon 2 , which encodes the highly conserved paired domain of $P A X 9$, and an absence of this change in the unaffected mother. Sequencing of 100 control individuals revealed an absence of the A259T alteration.

\section{Affected isoleucine residue is evolutionarily conserved across Pax 9 orthologs and related paralogs}

This novel PAX9 mutation is predicted to substitute phenylalanine for isoleucine at position 87 in the predicted protein sequence (Ile87Phe). Sequence comparisons indicate that the isoleucine residue is invariably conserved among the paired domains of related $P A X$ gene family members, as well as Pax9 homologs as distant as Drosophila (Figure 2).

Nuclear localization signal not disrupted by mutation To determine whether the mutation affects nuclear localization of the protein, COS7 cells were transfected with plasmids encoding Myc-tagged wild-type Pax9 or mutant Ile87Phe. Using immunocytochemistry, both proteins showed a predominantly nuclear expression 48-h post-transfection (Figure 3a). Similar results were obtained by cell fractionation and subsequent Western blot analysis, also $48 \mathrm{~h}$ after transfection (Figure $3 \mathrm{~b}$ ).

\section{Ile87Phe protein exhibits a specific loss of DNA binding}

Since the paired domain mediates DNA binding by PAX9, we performed EMSAs to determine effects on DNA-binding affinity. First, a Western blot of the GST-fusion proteins purified by affinity chromatography revealed that both proteins were intact and of the correct size. In our EMSA experiments, wild-type Pax9 efficiently retarded gel migration of ${ }^{32}$ P-labelled e5 and CD19-2(A-ins), two known paired domain-binding sites (Figure $4 \mathrm{~b}){ }^{7}$ In contrast, equivalent amounts of Ile87Phe displayed no affinity for the two elements. Additionally, the presence of increasing amounts of mutant protein with constant amounts of wildtype protein did not affect the ability of wild-type protein to bind DNA (Figure 4c). 
a

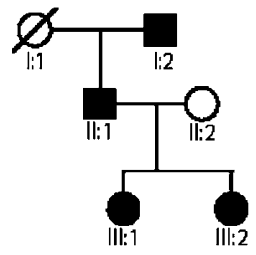

b

C
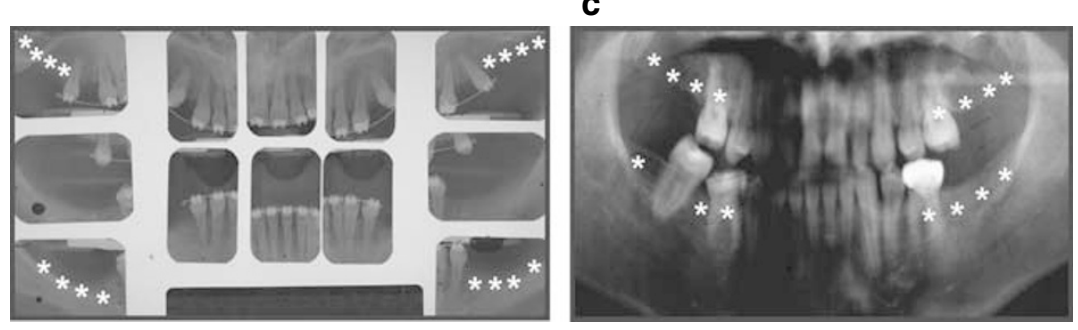

d

\begin{tabular}{|c|c|c|c|c|c|c|c|c|c|c|c|c|c|c|c|c|}
\hline \multirow[b]{3}{*}{ Maxilla } & \multicolumn{8}{|c|}{ Right } & \multicolumn{8}{|c|}{ Left } \\
\hline & \multicolumn{3}{|c|}{ M } & \multicolumn{2}{|c|}{$\mathbf{P}$} & C & \multicolumn{2}{|c|}{ I } & \multicolumn{2}{|c|}{ I } & \multirow{2}{*}{$\frac{\text { C }}{3}$} & \multicolumn{2}{|c|}{$\mathbf{P}$} & \multicolumn{3}{|c|}{$\mathbf{M}$} \\
\hline & 8 & 7 & 6 & 5 & 4 & 3 & 2 & 1 & 1 & 2 & & 4 & 5 & 6 & 7 & 8 \\
\hline Mandible & 8 & 7 & 6 & 5 & 4 & 3 & 2 & 1 & 1 & 2 & 3 & 4 & 5 & 6 & 7 & 8 \\
\hline \multirow{2}{*}{ III:1 } & * & * & * & * & & & & & & & & & * & * & * & * \\
\hline & * & * & * & * & & & & & & & & & * & * & * & * \\
\hline \multirow{2}{*}{ III:2 } & * & * & * & * & & & & & & & & & * & * & * & * \\
\hline & * & & * & * & & & & & & & & & * & * & * & * \\
\hline
\end{tabular}

Figure 1 Diagnosis of nonsyndromic, autosomal dominant posterior tooth agenesis. (a) Pedigree demonstrating autosomal dominant inheritance of tooth agenesis. (b, c) Panoramic radiograph of affected individuals lacking most permanent posterior teeth $(*)$. Note the difference in missing teeth in the two affected siblings. Patient III:2 has a lower right permanent second molar; the patient also has retained primary second molars in all quadrants. (d) Clinical phenotypes of affected proband and sibling. Stars represent congenitally missing teeth.

\section{Ile87Phe mutation does not affect interaction with Msx1}

To assess the ability of the mutant protein to complex with another transcriptional regulator in tooth development, Msx1, we carried out co-immunoprecipitation on extracts of COS7 cells transfected with epitope-tagged forms of Pax9 and Msx1. Expression of FLAG-tagged Msx1 with Myc-tagged wild-type or mutant Pax9 resulted in specific co-immunoprecipitation of Msx1 with both the wild type or mutant Pax9 proteins (Figure 5).

\section{Discussion}

In this report, we describe the identification of a novel missense mutation in the paired domain of PAX9 that results in the substitution of an evolutionarily conserved isoleucine residue by phenylalanine. The clinical phenotype of the family studied reflects a pattern of missing posterior teeth, primarily involving the molars. Based on our findings of a loss of DNA-binding ability by the mutant protein, we propose a loss-of-function mechanism to explain the resulting tooth agenesis.
The Ile87Phe substitution mutation affects the DNAbinding region of PAX9, the paired domain. X-ray crystal structure of the Drosophila PAIRED-DNA ${ }^{17}$ and PAX6 paired domain-DNA ${ }^{18}$ complexes indicates the paired box is a bipartite DNA-binding domain that is comprised of two distinct helix-turn-helix (HTH) motifs ( $(N$-subdomain (also known as PAI) and C-subdomain (also known as RED)) connected by a linker. Although the N-terminal PAI has been noted in some cases to be the most critical subdomain, another study provides compelling evidence that the recognition of DNA targets by Pax proteins is achieved through the coordinate use of both subdomains. ${ }^{19}$ Indeed, our demonstration of abrogation of DNA binding by a substitution mutation region within the C-subdomain supports the notion that this region plays a crucial role in the normal activities of the PAX9 paired domain.

Our analysis of the paired domains of other members of the $P A X$ gene family revealed another human genetic disorder caused by mutation of the equivalent Ile87 residue. In this case, a mutation involving a change from isoleucine to arginine (Ile87Arg) at the same position 


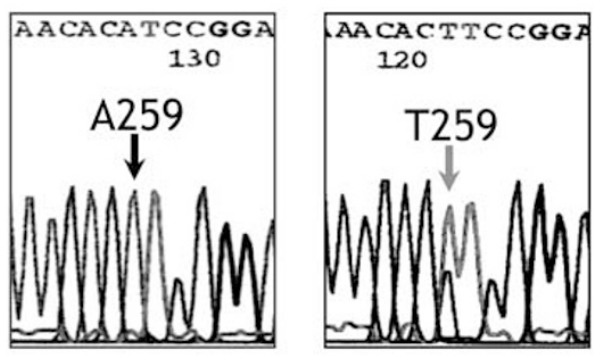

b

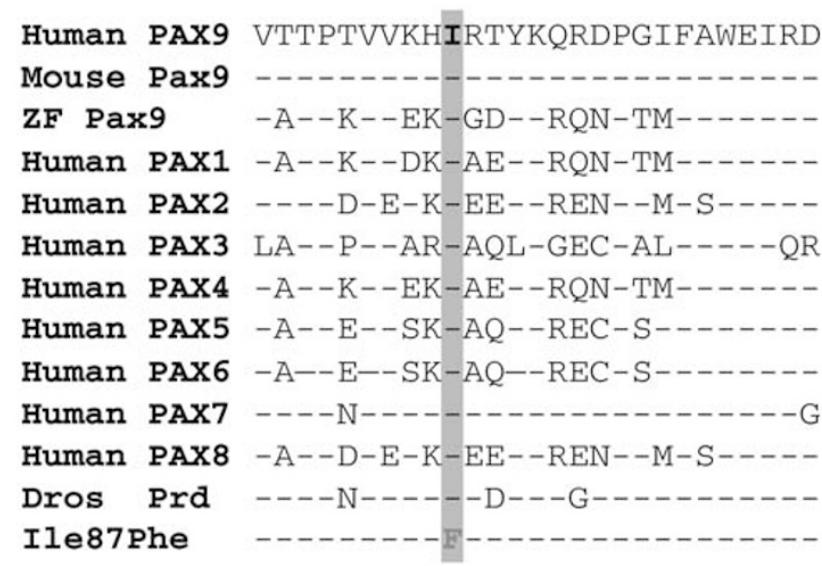

Figure 2 Posterior tooth agenesis is associated with a missense mutation in PAX9. (a) DNA sequence of paired-box domain of PAX9 in an affected family member (III: 1 ) showing a substitution mutation $(A \rightarrow T)$ at nucleotide 259 in exon 2. (b) The mutation causes an amino-acid change from an isoleucine to a phenylalanine at residue 87 (lle87Phe). Analysis of this region of exon 2 in PAX9 indicated that isoleucine is a highly conserved amino acid within the Pax family of genes and across species.
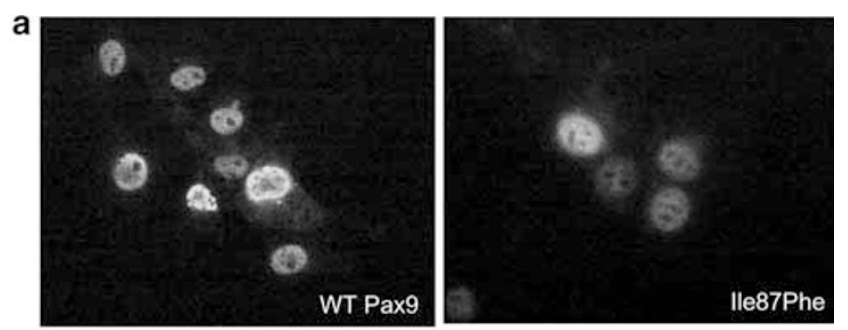

b

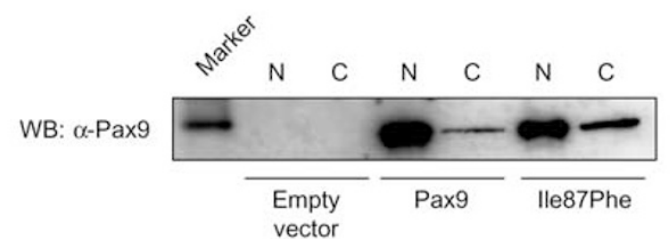

Figure 3 Nuclear localization unaffected by Pax9 mutation. (a) Nuclear localization of Myc-tagged wild-type Pax9 or lle87Phe Pax9, represented in green was similar when expressed in COS7 cells. (b) Western blot of nuclear $(\mathrm{N})$ and cytoplasmic (C) extracts obtained from transfected COS7 cells also shows wild-type and mutant proteins are primarily present in the nucleus. a

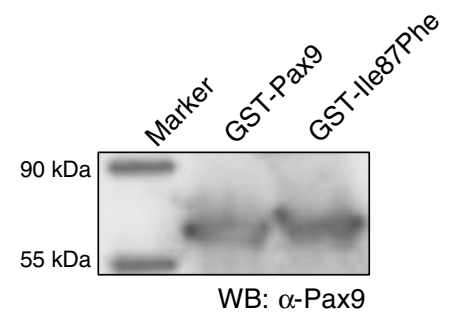

b

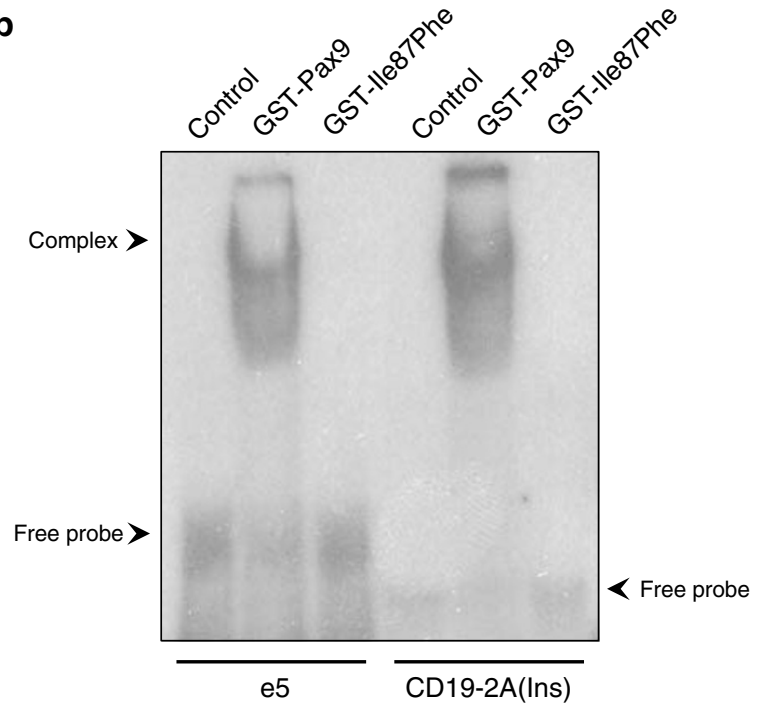

C

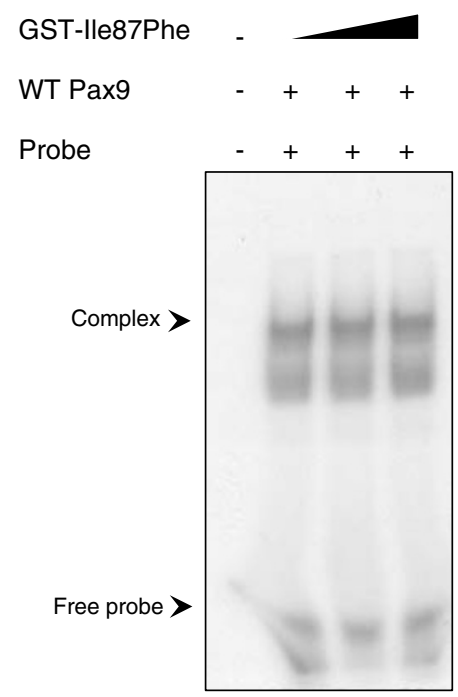

Figure 4 lle87Phe mutant is unable to bind paired domain recognition sequences and does not interfere with DNA-binding mediated by wild-type Pax9. (a) Western blot of purified recombinant GST-Pax9 and GST-lle87Phe Pax9 proteins. GST fusion proteins of the expected size $(\sim 63.5 \mathrm{kDA})$ are present. (b) Electromobility assay of two ${ }^{32}$ P-labelled high-affinity paired domain-binding sites with $100 \mathrm{ng}$ of wild-type Pax9 or lle87Phe reveals abolished DNA-binding activity of the mutant. (c) A fixed amount $200 \mathrm{ng}$ of GST-Pax9 was added to increasing amounts $(200,400,600 \mathrm{ng})$ of GST-lle87Phe Pax9. EMSA was performed using the CD19-2(A-ins) probe. 


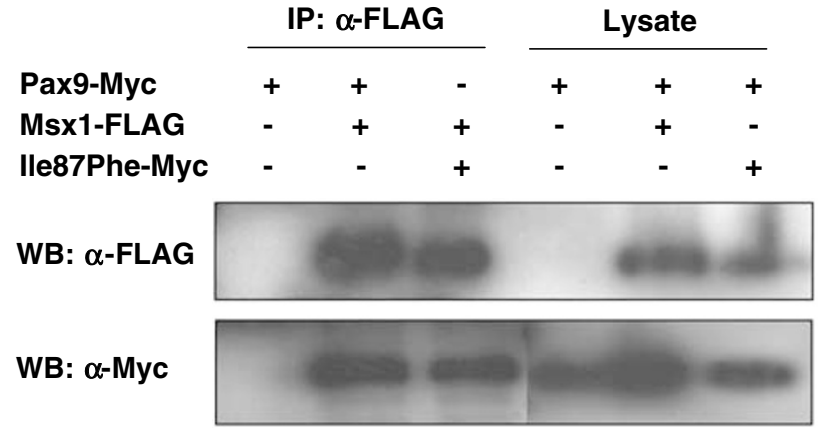

Figure 5 lle87Phe protein is able to physically interact with Msx1. Co-immunoprecipitation using extracts of COS7 cells coexpressing either Myc-Pax9 or Myc-lle87Phe and Flag-Msx1 expression vectors or the corresponding empty vector control. Equal amounts of proteins were immunoprecipitated with antibody to the Flag tag. The immunoprecipitated material was analyzed by Western blotting and both wild type and Pax9 are found only in the presence of Flag-Msx1. Additionally, cellular lysates were analyzed before co-immunoprecipitation to verify expression of Myc-Pax9 or Myc-lle87Phe and Flag-Msx1.

within the paired domain of PAX6 has been identified in a patient with aniridia. ${ }^{20}$ Functional studies showed the mutation dramatically reduced the DNA-binding ability of the paired domain, indicating that the carboxyl-terminal subdomain is essential for the in vivo function of the protein. The remarkable conservation of the isoleucine within the paired domain, coupled with the discovery of a mutation at the same site in a related gene is strongly supportive of the notion that Ile87Phe is causative in our family.

Sequence alignment of all known Pax proteins, including the Drosophila prd protein indicates Ile87 is an invariant residue. It lies in the fourth $\alpha$-helix of the paired domain within a hydrophobic pocket formed by Val81, Ile99, Leu103, Ile120, Val123, Leu124 and Leu127. The exact role of Ile87 is still unclear, but as it is not in the recognition helix of the HTH motif, it is thus likely to be important in protein conformation. ${ }^{18}$ Also, the change from isoleucine to phenylalanine represents a conservative substitution as both are nonpolar, hydrophobic amino acids. However, phenylalanine is considerably bulkier as it has a benzene ring. This supports the idea that the mutation disrupts the tight association of the amino acids that comprise the hydrophobic core, and thus the overall confirmation of the protein. However, we were unable to detect any effects on subcellular localization, indicating the mutation does not alter the nuclear localization signal, nor does it alter the structure of the protein to the extent where it cannot reenter the nucleus. Furthermore, we show that the Ile87Phe mutant protein is able to physically interact with the homeodomain-containing transcription factor Msx1. This suggests a region outside of the paired domain may be required for protein-protein interactions with Msx1.
Together, our results suggest that perturbation of DNA binding alone is sufficient for the pathogenesis of tooth agenesis.

Numerous studies suggest the $P A X 9$ mutant phenotype is dosage dependent: deletion of the PAX9 locus manifests as missing permanent teeth and the entire primary dentition; ${ }^{10}$ missense mutations result in oligodontia of only the permanent teeth; $;^{6,8,13,21}$ other mutations that would encode a truncated polypeptide present with missing permanent teeth, as well as some primary teeth. ${ }^{5,7-11}$ These findings, however, fail to fully explain the mechanisms underlying disease-causing mutations that result in less severe and variable phenotypes where not all posterior teeth are affected. Our functional analysis of the Ile87Phe protein demonstrated defects only at the level of DNA binding. Furthermore, we were unable to attribute a dominant-negative activity to the mutant protein, as the wild-type protein was able to efficiently bind DNA in the presence of the mutant protein. Taken together, our data suggest the combined activities of the wild-type and mutant alleles do not reach the threshold level necessary for normal tooth development.

Our assessment of the structural and functional defects produced by the Ile87Phe disease-causing mutation represents an initial step in explaining the pathogenic mechanisms underlying PAX9-mediated tooth agenesis. As more missense mutations are analyzed, it will become possible to correlate the position of a mutation, and the relative effect on function, with the observed developmental defects. This and future studies of naturally occurring paired domain mutations provides a paradigm for studies investigating the developmental function of this group of paired domain-containing transcription factors.

\section{Acknowledgements}

We gratefully acknowledge the support of the family and dentists who participated in this study. We also thank Dr Adele Schneider (Albert Einstein Medical Center) for her help in the recruitment of patients for our study. The assistance of Drs Puskas, Milewicz and Needleman (University of Texas Health Science Center at Houston Medical School, DNA Sequencing Core Facility) is also appreciated. We also thank Drs John Mensah and Amy Jackson for technical assistance and Adriana Cavender and Liza Shevchenko for assistance in preparation of the manuscript. This research was supported by the American Association of Orthodontists Foundation and UTHSC-Houston Faculty Development Funds to SAFB; The Texas Higher Education Coordinating Board grant, NIH Grant RO1 DE13668 to RDS and SAFB; NIH KO8DE14237 to HK, and UTHSCH UCRC (MO1RRO2558).

\section{References}

1 Peters H, Balling R: Teeth. Where and how to make them. Trends Genet 1999; 15: 59-65.

2 Jernvall J, Thesleff I: Reiterative signaling and patterning during mammalian tooth morphogenesis. Mech Dev 2000; 92: 19-29.

3 Thesleff I: Developmental biology and building a tooth. Quintessence Int 2003; 34: 613-620. 
4 Nik-Hussein NN: Hypodontia in the permanent dentition: a study of its prevalence in Malaysian children. Aust Orthod J 1989; 11: $93-95$.

5 Stockton DW, Das P, Goldenberg M, D'Souza RN, Patel PI: Mutation of PAX9 is associated with oligodontia. Nat Genet 2000; 24: 18-19.

6 Mostowska A, Kobielak A, Trzeciak WH: Molecular basis of nonsyndromic tooth agenesis: mutations of MSX1 and PAX9 reflect their role in patterning human dentition. Eur J Oral Sci 2003; 111: $365-370$.

7 Mensah JK, Ogawa T, Kapadia H, Cavender AC, D'Souza RN: Functional analysis of a mutation in PAX9 associated with familial tooth agenesis in humans. J Biol Chem 2004; 279: $5924-5933$.

8 Das P, Hai M, Elcock C et al: Novel missense mutations and a 288bp exonic insertion in PAX9 in families with autosomal dominant hypodontia. Am J Med Genet 2003; 118A: 35-42.

9 Frazier-Bowers SA, Guo DC, Cavender A et al: A novel mutation in human PAX9 causes molar oligodontia. J Dent Res 2002; 81: $129-133$.

10 Nieminen P, Arte S, Tanner D et al: Identification of a nonsense mutation in the PAX9 gene in molar oligodontia. Eur J Hum Genet 2001; 10: 743-746.

11 Klein ML, Nieminen P, Lammi L, Niebuhr E, Kreiborg S: Novel mutation of the initiation codon of PAX9 causes oligodontia. J Dent Res 2005; 84: 43-47.

12 Das P, Stockton DW, Bauer C et al: Haploinsufficiency of PAX9 is associated with autosomal dominant hypodontia. Hum Genet 2002; 110: 371-376.
13 Jumlongras D, Lin JY, Chapra A et al: A novel missense mutation in the paired domain of PAX9 causes non-syndromic oligodontia. Hum Genet 2004; 114: 242-249.

14 Czerny T, Schaffner G, Busslinger M: DNA sequence recognition by Pax proteins: bipartite structure of the paired domain and its binding site. Genes Dev 1993; 7: 2048-2061.

15 Goulding MD, Chalepakis G, Deutsch U, Erselius JR, Gruss P: Pax3 , a novel murine DNA binding protein expressed during early neurogenesis. EMBO J 1991; 10: 1135-1147.

16 Neubüser A, Koseki H, Balling R: Characterization and developmental expression of Pax9, a paired-box-containing gene related to Pax1. Dev Biol 1995; 170: 701-716.

$17 \mathrm{Xu} \mathrm{W}$, Rould M, Jun S, Desplan C, Pabo C: Crystal structure of a paired domain-DNA complex at a $2.5 \AA$ resolution reveals a structural basis for Pax developmental mutations. Cell 1995; 80: 639-650.

18 Xu E, Rould M, Xu W, Epstein J, Maas R, Pabo C: Crystal structure of the human Pax6 paired domain-DNA complex reveals specific roles for the linker region and carboxy-terminal subdomain in DNA binding. Genes Dev 1999; 13: 1263-1275.

19 Vogan KJ, Gros P: The C-terminal subdomain makes an important contribution to the DNA binding activity of the Pax3 paired domain. J Biol Chem 1997; 272: 28289-28295.

20 Tang HK, Chao LY, Saunders GF: Functional analysis of paired box missense mutations in the PAX6 gene. Hum Mol Genet 1997; 6: 381-386.

21 Lammi L, Halonen K, Pirinen S, Thesleff I, Arte S, Nieminen P: A missense mutation in PAX9 in a family with distinct phenotype of oligodontia. Eur J Hum Genet 2003; 11: 866-871. 